\title{
Comparison of SHR Mode IPL System with Alexandrite and Nd: YAG Lasers For Leg Hair Reduction
}

\author{
Şemsettin Karaca, Seval Doğruk Kaçar, Pınar Ozuğuz \\ Department of Dermatology, Faculty of Medicine, Afyon Kocatepe University, Afyonkarahisar, Turkey
}

\section{ABSTRACT}

Objective: Multiple lasers and light sources are currently available for hair removal and many studies comparing efficacy and safety exist. SHR mode IPL is a technique that gives the total energy in divided doses with fewer side effects. We compared a SHR mode IPL system with alexandrite and Nd: YAG lasers for leg hair reduction

Material and Methods: Twenty-five female participants with skin types II-IV, underwent treatment of unwanted hair on the cruris. Three selected areas were randomly treated with the SHR mode IPL system, Alexandrite laser and Nd: YAG laser in three sessions. Hair reduction was evaluated with digital photography by a blinded assessor every 6 weeks and 6 months after the last session. Pain severity, side effects and patient satisfaction analysis were also investigated.

Results: Twenty-one participants completed the trial. The mean hair reductions in 6 weeks after the last treatment were $50 \%$ for the IPL system, $53 \%$ for Alexandrite and 39\% for the Nd: YAG lasers. However after 6 months; $40 \%, 49 \%$ and $34 \%$ hair reduction was observed, respectively. The Alexandrite laser was the least painful system.

Conclusion: Although there is no obvious advantage of one laser system over the others in terms of treatment outcome, the Alexandrite laser is still the most efficient and reliable way of hair removal in fair skinned individuals.

Key Words: Hair removal, Alexandrite laser, Nd: YAG laser, SHR mode IPL system

Received: 26.02 .2012

Accepted: 02.05.2012

\section{Introduction}

Excess hair growth is a common problem for both genders. Up to now, many methods such as chemical depilation, shaving, waxing and plucking has been used for removal of unwanted hair. These methods can only have a short-term, transient effect. Although electrolysis can achieve permanent hair removal in experienced hands, it is inconvenient due to pain and prolonged treatment sessions $(1,2)$. The search for an efficient, practical method with minimum side effects and long lasting efficacy has led to the development of laser hair removal methods, in the last decade. Laser methods applied nowadays do not yet provide permanent removal; however they have gained popularity due to selective hair damage, decreased pain feeling, lesser time consumption, longer hair free interval and fewer side effects.

By using appropriate wavelength, pulse duration and fluence, thermal injury can be limited to the absorbing chromophore, melanin, which is rich in the hair bulb and shaft $(2,3)$. The interrogation point of progenitor stem cell destruction, which is hypothesized to be amelanotic, should be the major destination to achieve permanent hair removal (4). To overcome this situation, the modified theory of selective photothermolysis allows heat propagation to the surrounding tissue including the amelanotic stem cells by using longer pulse durations (5). As melanin has the highest density at the anagen phase and the hair growth center is more superficial at this phase, anagen hairs are more readily affected, so that multiple sessions with variable intervals are needed (3). In fact the portion of hair follicle which leads to permanent loss after destruction is still debated.

Long pulse wavelength $(600-1000 \mathrm{~nm})$ laser systems are preferred for epilation due to sufficient penetrance into a deeply located hair root. Thus, Ruby $(694 \mathrm{~nm})$, Alexandrite $(755 \mathrm{~nm})$, semi-conductor Diode $(810 \mathrm{~nm})$ and Nd: YAG (1064 $\mathrm{nm}$ ) laser systems are frequently employed for epilation from the short to longer wavelength spectrum. In addition, Intense pulse light (IPL) systems are used for epilation although they are not true laser systems $(1,3)$.

Currently, the Ruby laser is not used in hair removal due to hypopigmentations observed even in skin type II (6). Melanin absorption of normal mode Alexandrite $(755 \mathrm{~nm})$ lasers are very good. However, a few, postlaser pigmentation disorders are reported $(6,7)$. Due to longer wavelength, Nd: YAG (1064 $\mathrm{nm}$ ) lasers partially absorb melanin but deeper penetrance is achieved. In addition, it is safely utilized in dark-skinned pati- 
ents (1, 3). Epilation via IPL (590-1200 nm) sources using different filters are reported to be as successful as laser systems as a result of a thermal effect, with a risk of erythema, burn and pigmentation. IPL has the advantage of lower cost and larger spot size enabling more rapid treatment, but a greater number of treatment sessions are required to achieve satisfying results $(1,3,8)$.

Laser hair removal has become the most commonly used cosmetic procedure in the world. Multitechnology systems that encompass laser and light technologies in one platform are available, enabling greater versatility and flexibility in regard to therapeutic choices. As new devices are being developed, treatment of all skin types and all hair color is becoming possible. A super hair removal (SHR) mode for IPL system exhibits the total energy applied per session, counted in kilojoules, to a precise area with the advantage of fewer side effects than the usual IPL. Although many studies comparing the efficacy and side effects exist, triple studies are few and we did not find a study comparing Alexandrite, Nd: YAG and IPL systems. So, we aimed to compare the short wavelength Alexandrite laser $(755 \mathrm{~nm})$, the long wavelength $\mathrm{Nd}$ : YAG laser $(1064 \mathrm{~nm})$ and the wide wavelength IPL (650-950 nm), all in one platform, in terms of efficacy and side effects.

\section{Material and Methods}

\section{Participants}

This is a prospective, single centered study approved by the Afyon Kocatepe University Medical Ethics committee and conformed to the guidelines of the Declaration of Helsinki. 25 untanned volunteer Turkish women with Fitzpatrick skin types II to IV and dark brown to black hair were recruited to the study. The detailed procedure, expected results and complications were discussed with the participants and all of the participants signed the informed consent. Exclusion criteria included any previous laser or electrolysis treatment, usage of waxing or depilation within the previous two months, history of isotretinoin treatment or photosensitivity and hormonal dysfunction.

\section{Procedure}

Each participant underwent a series of 3 sessions at 6 week intervals. Follow up evaluations done at 6 weeks after each session and 6 months after the final session. An imaginary line was drawn from the tibial tuberosity down to the third foot finger to both anterior crural regions. To the lateral side of the right tibial tuberosity, measuring $2 \mathrm{~cm}$ down from the knee, a square is drawn keeping the imaginary line at one edge, another square is drawn from the inferior medial corner to the left of this line, again keeping it at one edge. A third square is drawn to the medial side of the left tibial tuberosity (Figure 1). These $10 \mathrm{~cm}$ edged squares were treated in a randomized manner using clockwise rotation with each participant. Lasers and intense pulsed light systems used in this study, which are on the same platform (Harmony-XL; Alma Lasers Ltd., Caesarea, Israel), were as follows (Table 1):

1. A 755-nm Alexandrite laser with a round spot size of $5 \mathrm{~mm}$, maximum fluence of $32 \mathrm{~J} / \mathrm{cm}^{2}$, pulse frequency of $2-4 \mathrm{~Hz}$.
2. A 1064-nm Nd:YAG laser with a round spot size of $6 \mathrm{~mm}$, adjustable fluences of $30-150 \mathrm{~J} / \mathrm{cm}^{2}$, variable pulse duration of 45 to 60 milliseconds.

3. A $650-950 \mathrm{~nm}$ Advanced Fluorescence Technology (AFT ${ }^{\mathrm{TM}}$ ) light based system with a rectangular spot size of 3 versus $1 \mathrm{~cm}$, fluence of $1-7 \mathrm{~J} / \mathrm{cm}^{2}$, pulse frequency of 1.3 and 30 seconds and a contact cooling mechanism was attached. A thin layer of ultrasound gel was applied to the treatment area as recommended by the manufacturer. For large areas such as legs operation time intervals are set as 30 seconds and at this interval the in-motion technique is used, which requires continuous motion giving adjusted total energy for a particular area.

The set up and fluences used in the three systems were within the recommended range based on skin type by the manufacturer (Table 2). All treatments were performed by the same operator. The performing operator was not involved in assessment. Participants were instructed not to remove hair 10 days before the follow up visits for accurate assessment. No other hair removal method other than shaving was permitted between sessions and up to the last follow up. After assessment, the treatment sites were shaved before the procedure. Ice packs were used before and after laser treatments to reduce adverse effects and pain. Local anesthesia was not applied. Standard safety measures were taken during the laser procedure.

Pretreatment and follow up photographs were taken using a digital camera (Canon EOS DRXSi/EOS 450D, 12.2 megapixel image sense digital camera) using identical lightning, pati-

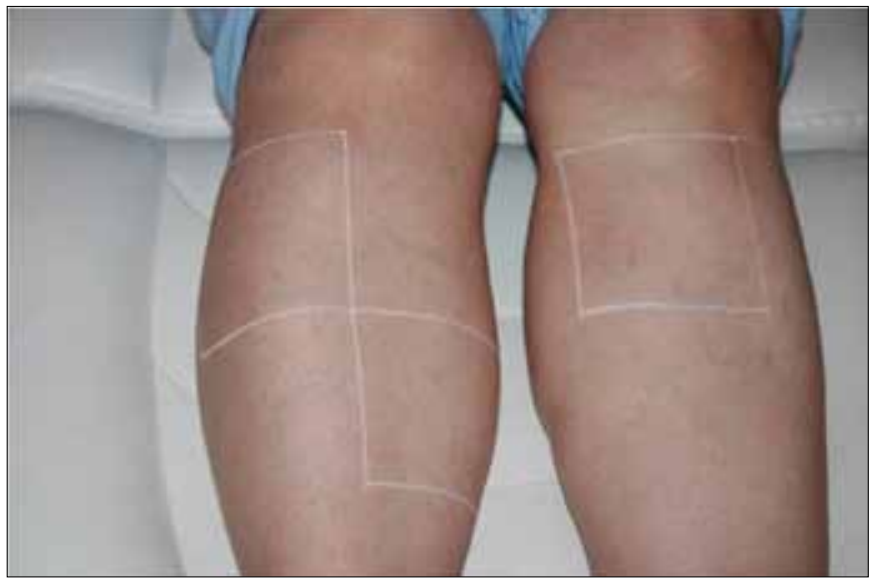

Figure 1. The schematic view of three treatment areas

Table 1. The features of laser device (Harmony-XL; Alma Lasers Ltd., Caesarea, Israel)

\begin{tabular}{|lccc|}
\hline Laser type & Alexandrite & Nd: YAG & $\begin{array}{c}\text { IPL } \\
\text { (SHR mode) }\end{array}$ \\
\hline Wavelength $(\mathrm{nm})$ & 755 & 1064 & $650-950$ \\
Spot size & $5 \mathrm{~mm}$ & $6 \mathrm{~mm}$ & $3 \times 1 \mathrm{~mm}$ \\
Pulse width & $2 \mathrm{~ms}$ & $45-60 \mathrm{~ms}$ & $30 \mathrm{sec}$ \\
Fluences $\left(\mathrm{J} / \mathrm{cm}^{2}\right)$ & $1-32$ & $30-150$ & $1-7$ \\
Cooling & none & none & Contact cooling \\
\hline
\end{tabular}


Table 2. The recommended fluence ranges of the three systems

\begin{tabular}{|c|c|c|c|c|}
\hline & & $\begin{array}{c}\text { Alexandrite } \\
\text { (6 mm spot size) }\end{array}$ & $\begin{array}{c}\text { Nd:YAG } \\
\text { (5 mm spot size) }\end{array}$ & $\begin{array}{c}\text { IPL } \\
(3 \times 1 \mathrm{~cm} \text { spot size } / \\
100 \mathrm{~cm}^{2} \text { total area) }\end{array}$ \\
\hline \multicolumn{5}{|c|}{ Skin type } \\
\hline II & $\begin{array}{l}\text { Fluence } \\
\text { Pulse width }\end{array}$ & $\begin{array}{c}25-30 \mathrm{~J} / \mathrm{cm}^{2} \\
2 \mathrm{~ms}\end{array}$ & $\begin{array}{c}80-100 \mathrm{~J} / \mathrm{cm} 2 \\
45-60 \mathrm{~ms}\end{array}$ & $\begin{array}{c}7 \mathrm{~J} / \mathrm{cm}^{2}(2.0-2.6 \mathrm{~kJ}) \\
30 \mathrm{sec}\end{array}$ \\
\hline III & $\begin{array}{l}\text { Fluence } \\
\text { Pulse width }\end{array}$ & $\begin{array}{c}22-24 \mathrm{~J} / \mathrm{cm}^{2} \\
2 \mathrm{~ms}\end{array}$ & $\begin{array}{c}80-100 \mathrm{~J} / \mathrm{cm} 2 \\
45-60 \mathrm{~ms}\end{array}$ & $\begin{array}{c}7 \mathrm{~J} / \mathrm{cm}^{2}(2.0-2.6 \mathrm{~kJ}) \\
30 \mathrm{sec}\end{array}$ \\
\hline IV & $\begin{array}{l}\text { Fluence } \\
\text { Pulse width }\end{array}$ & $\begin{array}{c}18-21 \mathrm{~J} / \mathrm{cm}^{2} \\
2 \mathrm{~ms}\end{array}$ & $\begin{array}{c}80-100 \mathrm{~J} / \mathrm{cm} 2 \\
45-60 \mathrm{~ms}\end{array}$ & $\begin{array}{c}6 \mathrm{~J} / \mathrm{cm}^{2}(2.0-2.6 \mathrm{~kJ}) \\
30 \mathrm{sec}\end{array}$ \\
\hline
\end{tabular}

Table 3. Hair reduction analysis

\begin{tabular}{|c|c|c|c|c|}
\hline & \multirow[b]{2}{*}{ Hair reduction } & \multicolumn{3}{|c|}{ Wilcoxon signed ranks test } \\
\hline & & $\begin{array}{l}\text { Alexandrite } \\
\text { versus IPL }\end{array}$ & $\begin{array}{c}\text { Alexandrite } \\
\text { versus } \\
\text { Nd:YAG }\end{array}$ & $\begin{array}{c}\text { IPL } \\
\text { versus } \\
\text { Nd:YAG }\end{array}$ \\
\hline \multicolumn{5}{|c|}{6 weeks after three sessions } \\
\hline Alexandrite & $53 \%$ & NS $(p=0.244)$ & $S(p<0.05)$ & $S(p<0.05)$ \\
\hline IPL & $50 \%$ & & & \\
\hline $\mathrm{Nd}: Y A G$ & $39 \%$ & & & \\
\hline \multicolumn{5}{|c|}{6 months after three sessions } \\
\hline Alexandrite & $49 \%$ & $S(p=0.002)$ & $S(p=0.001)$ & NS $(p=0.06)$ \\
\hline IPL & $40 \%$ & & & \\
\hline $\mathrm{Nd}: Y A G$ & $34 \%$ & & & \\
\hline
\end{tabular}

ent positioning and camera settings by the same investigator. Hair counts from digital photographs were performed on a $100 \mathrm{~cm}^{2}$ area in all three sites. The investigator taking photographs also assessed the hair count and was blinded to the treatment applications.

In every session, participants marked visual analogue scale (VAS) for pain with a range from 0 (no pain) to 10 (unbearable pain) and the immediate side effects were evaluated. Participants also self assessed the procedure, measuring improvement and satisfaction based on a scale ranging from 0 (no improvement) to 100 (total disappearance) at the end of three sessions and at the $6^{\text {th }}$ month follow up.

Data were recorded and analyzed using the SPSS statistical analysis software version 18. A $p$ value of 0.05 or less was considered significant. The percentage reduction in hair counts in all three sites compared using the Friedman test and Wilcoxon signed rank tests were used to compare the laser systems.

\section{Results}

Of the twenty five volunteers, twenty one participants (mean age 32.85 years, range between 25-45y) completed the study. Two of them were withdrawn from the study due to pregnancy, and the remaining two participants due to nonattendance at follow ups. A total of $19.0 \%$ (4 subjects), $52.4 \%$ (11 subjects) and $28.6 \%$ (6 subjects) of participants have skin photo types II, III and IV, respectively.

There was no statistically significant difference in pretreatment hair counts among the three sites; for the IPL site, $390 \pm 73$ (mean \pm SEM), f Alexandrite site $384 \pm 88, \mathrm{Nd}$ : YAG site $388 \pm 71(p=0.172)$. All three systems resulted in long term hair reduction.

At the end of three sessions, the reduction in hair count was $53 \%, 50 \%$ and $39 \%$ for Alexandrite, IPL and Nd: YAG systems, respectively. The decrease in hair count for Alexandrite and IPL systems were statistically significantly greater than that observed in $\mathrm{Nd}$ : YAG laser (for both, $\mathrm{p}<0.05$ ). Later, after 6 months the reduction in hair count was decreased to $49 \%$, $40 \%$ and $34 \%$ in the same order, again. This time the decline in hair count for Alexandrite laser was statistically significantly greater than that observed in IPL and Nd: YAG systems (for both, $\mathrm{p}<0.05)$ (Table 3 ). The reduction in hair count was not statistically related to skin type for any system.

Interpreting the mean VAS score for pain for all three sessions, the Alexandrite laser system was the least painful with a mean pain score of 3.90, followed by the IPL system (5.71) and Nd: YAG systems (6.95). Statistical analysis showed a sig- 
nificant pain related to $\mathrm{Nd}$ : YAG and IPL systems but there was no significant difference between two systems.

Patient satisfaction analysis at the end of three sessions showed a mean score of 33 for IPL system, 37 for Alexandrite laser and 27 for Nd: YAG laser, which are quite similar. Statistically the Alexandrite system showed better satisfaction rates than Nd: YAG and IPL (for both, $\mathrm{p}<0.05$ ). 6 months after treatments the scores were higher than expected, in same order as 39, 46 and 38. Again, Alexandrite laser satisfaction scores were higher than IPL and Nd: YAG $(p<0.05)$.

There was only mild temporary erythema and perifollicular edema after some treatment sessions. However, none of the participants experienced pigmentation alterations, vesiculation, burns, infection or scarring.

\section{Discussion}

The patient's understanding of permanent hair removal frequently implies lifelong sustained no hair regrowth. In fact, permanent reduction rather than removal is the usual outcome (3). Detailed explanation about expectation and proper patient selection is crucial to achieve higher satisfaction rate. On the other hand, knowing the capability of laser systems is another critical issue. Several laser and light based devices are in use for laser hair removal. There are many comparative studies on the treatment outcome and complications between different systems. Although a trial comparing Alexandrite, Nd: YAG and IPL systems all together has not yet been found, studies comparing pair by pair I are found, usually revealing Alexandrite as the most effective photoepilation method. Similarly, our study showed the Alexandrite system most efficient so that, even six months after the last session, nearly $50 \%$ hair clearance is achieved. In a study comparing Alexandrite, diode and $\mathrm{Nd}$ : YAG lasers after three treatments, the reduction in hair was $70.3 \%, 59.7 \%$ and $47.4 \%$ respectively (9). In addition, similar to our study, Alexandrite laser was reported as best tolerated, whereas Nd: YAG system was the most painful application. A similar comparison trial with long pulsed versions of Alexandrite, diode and Nd: YAG lasers systems revealed $65.6 \%, 46.9 \%$ and $42.4 \%$ mean hair reduction, respectively (10). In another work, the mean hair reduction with Alexandrite system was $75.9 \%$ and with $\mathrm{Nd}$ : YAG system $73.6 \%$ after four sessions (11). On the contrary, they have reported higher pain severity in the Alexandrite group. In a split-face study, the decrease in hair counts was $46 \%$ in the Alexandrite treated site and $27 \%$ in the IPL treated site (12). The specific wavelength and short pulse duration of laser and split pulse delivery in IPL were indicated as a probable cause. Another study comparing short-pulse and long pulse Alexandrite lasers with IPL systems that have different cut-off filters showed no superior efficacy of one to the other but the Alexandrite system was found to be associated with higher side effects (13). In a comparison trial by Amin and Goldberg (14), which evaluated the efficacy of an IPL device (red filter), an IPL device (yellow filter), a $810 \mathrm{~nm}$ diode laser, and a $755 \mathrm{~nm}$ Alexandrite laser, hair counts at 1, 3 and 6 months after the second treatment showed almost $50 \%$ reduction in all devices without any superiority of one to other.
Interestingly, at the end of the follow up period revealing the long term efficacy, the reduction with IPL system declined significantly, showing that the immediate effect was temporary rather than permanent. On the other hand, a delayed increased response with $\mathrm{Nd}$ : YAG system is achieved that was nearly equal to the IPL response. In a study by Goh (15), after a single session, long pulsed Nd: YAG lasers was found to be more effective than IPL therapy for hair removal in skin types IV-VI, with fewer side-effects. The author explained this with the long pulse Nd: YAG laser being able to penetrate 5-7 $\mathrm{mm}$ depth into the dermis in contrast to the short wavelength IPL system used in this study. Recently in a comparative study of Nd: YAG and IPL systems in dark skinned individuals, the $\mathrm{Nd}$ : YAG system was found most efficient after five treatments (16).

Laser parameters seem to be important in choosing the ideal laser for the patient. With lower fluences, temporary removal rather than permanent is achieved. Roosen et al. (17) studied the effect of low fluence photoepilation on hair follicles with findings suggesting catagen transition of anagen hair follicles. Long term hair removal largely depends on hair color, skin color and the tolerated fluence. It is said that when optimal parameters are used, each treatment session leads to about $15-30 \%$ hair loss $(1,3)$. In this study, six months after three sessions, only one third of initial hair was completely lost with the Nd: YAG system. This result may be related to the number of sessions, as emphasized by Lorenz et al. (18) in their study that at least four to five sessions are necessary for an acceptable result with the $\mathrm{Nd}$ : YAG laser. Besides the fluences in our study as recommended by the manufacturer, neither a test dose nor the maximum tolerated dose is aimed for. Even perifollicular edema and erythema was noted in most of the patients and no adverse effects were observed. Thus, the fluences may be lower than the level that would be tolerated. On the other hand, Sadick et al. (19) did not find a positive correlation between hair reduction and number of treatments for IPL.

The effectiveness of different optical devices for hair removal varies significantly among patients due to anatomical location, skin type, hair color, hair thickness, duration of the hair follicle cycle and androgen status. This study is designed as within participant comparative study to eradicate these variables. Although differences between individuals exist, about $62-88 \%$ of the hair in the lower leg are in the telogen phase, whereas the duration of the resting period for hair follicles is about 3-6 months (3). Depending on this data, we have decided on a 6 months follow up period after the end of three sessions. After six months, nearly all participants had some hair regrowth that make us consider whether a longer followup for a reliable assessment of long term effects should be planned.

Cold packs, the least effective type of cooling, are used in this study without any topical anesthesia, to objectively evaluate pain. The results were similar to literature findings, pointing to the Alexandrite laser as the most painless system, despite the contact cooling system attached to the IPL probe. Furthermore, pain scores with IPL were nearly equal to pain scores for the Nd: YAG laser. This is a conflicting result as the SHR mode IPL is thought to have the advantage of less pain and side effects. 
The low number of participants and the treatment sessions can be accepted as our limitations as well as not determining the maximum tolerated dose. The usage of a device with three systems in one platform can also be regarded as a limitation.

\section{Conclusion}

Although there is no obvious advantage of the SHR mode IPL system over the others in terms of treatment outcome, the Alexandrate laser is still the most efficient and painless light source in comparison with the Nd: YAG and IPL of the SHR mode. Despite the continuous efforts for the development of new laser systems in permanent hair removal with a minimal number of session, we can state that there is as yet no developed safe and efficient light source that meets the expectations.

\section{Conflict of Interest}

This study was supported by Afyon Kocatepe University, scientific fund (09.TIP.01)

\section{References}

1. Ibrahimi OA, Avram MM, Hanke CW, Kilmer SL, Anderson RR. Laser hair removal. Dermatol Ther 2011;24:94-107. [CrossRef]

2. Gorgu M, Aslan G, Akoz T, Erdogan B. Comparison of Alexandrite laser and electrolysis for hair removal. Dermatol Surg 2000;26:37-41. [CrossRef]

3. Goldberg DJ. Laser hair removal. In: Goldberg DJ. ed. Laser dermatology: pearls and problems. Blackwell Publishing 2008:37-61.

4. Comma S, Gallardo O, Bernard BA. The human hair follicle contains two distinct $\mathrm{K} 19$ positive compartments in the outer root sheath: A unifying hypothesis for stem cell reservoir? Differentiation 2000; 66:157-64. [CrossRef]

5. Altshuler GB, Anderson RR, Manstein D, Zenzie HH, Smirnov MZ. Extended theory of selective photothermolysis. Lasers Surg Med 2001;29:416-32. [CrossRef]

6. Sadighha A, Mohaghegh Zahed G. Meta-analysis of hair removal laser trials. Lasers Med Sci 2009;24:21-5. [CrossRef]

7. Nanni CA, Alster TS. Laser assisted hair removal: side effects of Q-switched Nd:YAG, long-pulsed ruby and alexandrite lasers. J Am Acad Dermatol 1999;41:165-71. [CrossRef]
8. Haedersdal M, Wulf HC. Evidence-based review of hair removal using lasers and light sources. JEADV 2006;20:9-20. [CrossRef]

9. Khoury JG, Saluja R, Goldman MP. Comparative evaluation of long-pulse alexandrite and long-pulse $\mathrm{Nd}$ : YAG laser systems used individually and in combination for axillary hair removal. Dermatol Surg 2008;34:665-7. [CrossRef]

10. Bouzari N, Tabatabai H, Abbasi Z, Firooz A, Dowlati Y. Laser hair removal: comparison of long-pulsed Nd: YAG, long-pulsed alexandrite, and long-pulsed diode lasers. Dermatol Surg 2004;30:498-502. [CrossRef]

11. Davoudi SM, Behnia F, Gorouhi F, Keshavarz S, Nassiri Kashani M, Rashighi Firoozabadi M, et al. Comparison of long-pulsed alexandrite and Nd:YAG lasers, individually and in combination, for leg hair reduction: an assessor-blinded, randomized trial with 18 months of follow-up. Arch Dermatol 2008;144:1323-7. [CrossRef]

12. McGill DJ, Hutchison C, McKenzie E, McSherry E, Mackay IR. A randomised, split-face comparison of facial hair removal with the alexandrite laser and intense pulsed light system. Lasers Surg Med 2007;39:767-72. [CrossRef]

13. Marayiannis KB, Vlachos SP, Savva MP, Kontoes PP. Efficacy of long- and short pulse alexandrite lasers compared with an intense pulsed light source for epilation: a study on 532 sites in 389 patients. J Cosmet Laser Ther 2003;5:140-5. [CrossRef]

14. Amin SP, Goldberg DJ. Clinical comparison of four hair removal lasers and light sources. J Cosmet Laser Ther 2006;8:65-8. [CrossRef]

15. Goh CL. Comparative study on a single treatment response to long pulse Nd:YAG lasers and intense pulse light therapy for hair removal on skin type IV to $\mathrm{Vl}$--is longer wavelengths lasers preferred over shorter wavelengths lights for assisted hair removal. J Dermatolog Treat 2003;14:243-7. [CrossRef]

16. Ismail SA. Long-pulsed Nd:YAG laser vs. intense pulsed light for hair removal in dark skin: a randomized controlled trial. Br J Dermatol 2012;166:317-21. [CrossRef]

17. Roosen GF, Westgate GE, Philpott M, Berretty PJM, Nuijs T, Bjerring $\mathrm{P}$. Temporary hair removal by low fluence photoepilation: Histological study on biopsies and cultured human hair follicles. Lasers Surg Med 2008;40:520-8. [CrossRef]

18. Lorenz S, Brunnberg S, Landthaler M, Hohenleutner U. Hair removal with the long pulsed Nd:YAG laser: a prospective study with one year follow-up. Lasers Surg Med 2002;30:127-34. [CrossRef]

19. Sadick NS, Weiss RA, Shea CR, Nagel H, Nicholson J, Prieto VG. Long-term photoepilation using a broad-spectrum intense pulsed light source. Arch Dermatol 2000;136:1336-40. [CrossRef] 